\title{
CONTACT LENS WEAR AND THE OXYGEN ISSUE: A REVIEW
}

\author{
BY \\ IWUAGWU, F.O. \\ DEPARTMENT OF OPTOMETRY, \\ IMO STATE UNIVERSITY, OWERRI, \\ IMO STATE, NIGERIA \\ Email:frankiwuagwu@yahoo.com
}

\begin{abstract}
A review on contact lenses (CLs) and the importance of oxygen permeability (DK) and transmissibility $(D K / L)$ in contact lens $(C L)$ wear was undertaken. Motivation is a primary factor in determining a good candidate for CLs. Ocular consideration; systemic consideration and non-compliance of patients limit a patient's suitability for $C L$ wear. The majority of complications encountered with $C L$ wear are manageable by discontinuing their use. More severe, vision-threatening complications are rare. Hypoxia contributes most to the complications of $C L$ wear. Minimization of extended or over night-wear along with attention to compliance in lens care reduces the risk of complications associated with CL wear.
\end{abstract}

KEYWORDS: Contact lenses, Oxygen transmissibility, Rigid gas permeable lenses, Hydrogel contact lenses

\section{INTRODUCTION}

The most common reason patients seek ophthalmic care is to optimize visual acuity. Estimates suggest that about $50 \%$ of the population utilize some form of refractive correction ${ }^{1}$, and the natural history of presbyopia indicates that virtually everyone, who lives long enough, will benefit from one form of optical correction or the other.

The use of contact lenses (CLs) to neutralize refractive error has lasted for over 100years, but clinical success in the use of $\mathrm{Cls}$ for visual correction came much later. The original CLs were almost exclusively of large or haptic design, and all were made from glass. Feinbloom made a scleral CL with glass optics and a plastic carrier in the late 1930s, but the first practical plastic (polymethyl methacrylate or PMMA) CL was developed by Tuohy in the late 1940s. Hydrogel CLs were invented by Wichterle in the late 1950s. In the 1970s, after recognition of the role of corneal oxygenation in achieving physiological tolerance, hydrogel CLs with enhanced oxygen transmissibility and rigid gas permeable (RGP) CLs became available. These advances and other improvements in both materials and designs have resulted in CLs that are applicable for most forms of refractive errors and are both safe and effective for most patients ${ }^{2}$.

A random sampling of some major C. L. Clinics in Nigeria suggests that approximately, out of about $100,000(0.1 \%)$ Nigerians who use CLs, the vast majority (about 95\%) wear hydrogel CLs.
The majority of complications encountered with daily wear CLs are manageable by discontinuing their use. Inconvenience, minor physiological and allergic problems and interruptions in wear are commonplace.

More severe, vision-threatening complications are less common and include corneal microbial infections $^{3}$ and extreme forms of corneal neovascularization ${ }^{4}$, which can lead to scarring of the cornea in the area of the visual axis. The incidence of cornea microbial infection is about 1 case per 1000 wearers per year, ${ }^{5,6}$. Extending CL wear through one or more sleep cycles appears to increase both the prevalence and severity of all complication $^{7,8}$.

About $33 \%$ of the complications encountered with contact lens wear are attributable to hypoxia, and thus the oxygen transmissibility of a contact lens is probably the most important single parameter in terms of maintaining the physiologic integrity of the cornea'. Allergic and toxic effects are uncommon if a regular lens replacement regimen and modern lens care solutions are used.

Many factors help determine whether a patient is a good candidate for CLs or not. Primary among these is motivation to be a successful CL wearer. Unfortunately, there is no singular test or battery of tests that can predict success in wearing CLs.

\section{DISCUSSION}

Some factors that suggest whether a patient is a good candidate for CL wear involve optical, physiologic, and cosmetics considerations. 
Contact lenses improve visual function by neutralizing ametropia, or minimizing distortion, especially when the patient suffers from more than a modest spherical refractive error or astigmatism. Myopic patients benefit from CLs, compared with their spectacle corrections. The reverse is true for both hyperopic and aphakic patients; however, such patients benefit from enhanced field of vision with $\mathrm{CLs}^{10}$. For anisometropic patients, aniseikonia and prismatic effects may be reduced or eliminated with CL wear.

Although many patients with presbyopia wear CLs, presbyopia is not specially an indication for CL correction. Presbyopic patients may wear distance CLs and use additional reading spectacles of various types to address their presbyopia. Most presbyopes often use mono-vision correction, in which one eye wears a CL to correct for distance vision and the other wears a CL to correct for near vision. Various bifocal CLs are available in either RGP or hydrogel materials.

Aphakia and binocular vision problems especially accommodative esotropia and convergence excess can be managed with the use of CLs ${ }^{11,12}$. Rigid CLs can provide optically smooth anterior corneal surface made irregular by disease (example keratoconus or corneal microbial infection), trauma, or surgery (example penetrating keratoplastic or ineffective refractive surgery). Hydrogel lenses are used as ophthalmic bandages ${ }^{13}$ following corneal trauma or refractive corneal surgery. Rigid CLs can be used to manage ${ }^{14}$ or reduce $^{15}$ myopia. Correcting ametropia by placing a lens directly on the corneal surface improves cosmesis by eliminating the need for a spectacle frame and often- unattractive corrective ophthalmic lenses. Some patients elect to wear colored CLs simply to change the appearance of their eye color. Opaque CLs also may be used for their prosthetic effect (like, masking an unattractive corneal scar or damaged iris or providing an artificial pupil in the treatment of aniridia) ${ }^{16}$.

Some factors limit a patient's suitability for CL wear. These include ocular considerations, systemic considerations and noncompliance of patients.

CL wear should be approached cautiously with patients who present with any active anterior segment disease, especially ocular (or adnexial) inflammation, infection, or severe dry eye conditions, because of the possible increased risk of complications, especially corneal neovascularisation (NV) or infection. Such diseases include acne rosacea, Sjogren's syndrome, atopic dermatitis, corneal exposure, severe blepharitis, conjunctival cicatrisation, neurotrophic keratitis, dacryocystitis, and patent filtering blebs. Therapeutic contact lenses are occasionally used as bandages.

Placing the lens directly in the precorneal tear film increases the risk of tissue compromise. CL use should therefore be approached cautiously for either the monocular patient (because of risk to the patient's only useful eye) or for the patient who is engaged in an avocation or vocation with exposure to a particularly dirty or dry environment. Such individuals may be advised to wear protective spectacles.

An abnormal tear layer, whether insufficient in volume or of poor quality, decreases the likelihood of successful and asymptomatic CL wear, but CLs should be considered in the context of patient's motivation and other relevant indications. Some mild forms of abnormal tear layers can be treated with supplemental artificial tear drops or ointments and plugging of the puncta.

The patient's inability to manipulate and care for CLs adequately should be handled with caution. Patients suffering from immunosuppressive disease (e.g. AIDS), rheumatoid arthritis, or diabetes, which may lead to insufficient lacrimation or increased risk for corneal NV and infection, should also be treated with caution ${ }^{17,18}$. Practitioners should exercise caution and occasionally exercise restraint, when considering CL fitting for patients whom they know or suspect will not comply with appropriate CL care and general hygiene as to place themselves at increased risk for severe complications.

\section{TYPES OF CONTACT LENSES}

Contact lenses can be classified into two main broad categories: soft (hydrogel) and rigid contact lenses. These CLs are available in a wide variety of parameters for both spherical and spherocylindrical corrections. There are also several ñ́hybridòCLdesigns and materials.

Spherical hydrogel CLs are indicated for the correction of myopia and hyperopia when astigmatism is limited to less than 1.00 diopter ${ }^{19,20}$. The U.S. Food and Drug Administration (FDA) has classified all hydrogen materials into four groups ${ }^{21}$, which are believed to behave the same (way) chemically. Oxygen permeability (DK) of the hydrogel materials in all groups increases with water content $\mathrm{t}^{22}$. Oxygen transmissibility (DK/L) is lens specific, and therefore depends on both the 
water content, the DK, and the thickness profile of the lens $(\mathrm{L})^{23-26}$. Another type of hydrogel $\mathrm{CL}$ material, in which silicone (for enhanced DK) is blended with hydrogel material (for comfort), is also available ${ }^{27}$. Toric hydrogel lenses ${ }^{28-30}$ are indicated for patients who have astigmatism greater than $0.75 \mathrm{D}$. Standard designs frequently correct astigmatism up to $8.00 \mathrm{D}$ of astigmatism. Toric hydrogel lenses are more expensive than the spherical designs, and may not provide universally stable visual results ${ }^{31}$.

Variable optical results and comfort levels may occur in patient's who have insufficient tears with all types of hydrogel CLs, especially toric lenses. On the other hand, severe previous limbal desiccation at the $3 \mathrm{O}^{\prime}$ clock and $9 \mathrm{O}^{\prime}$ clock positions (3/9 O'clock staining) from the use of rigid CLs, with or without subsequent superficial neovascularisation, is an indication for fitting both spherical and toric hydrogel CLs in patients with adequate tears $^{32}$.

Rigid CLs usually provide better visual results than do hydrogel CLs in situations of either regular or irregular astigmatism of the corneal surface. Insufficient tear will not affect the optics of rigid CLs (depends on the type specified or provide reference), but this condition increases the prevalence of both intolerance and some physiological complications in rigid CLs.

RGP materials are available in a wide range of powers $\mathrm{O}_{2}$ permeability ${ }^{33}$, plastic hardness, wettability, and specific gravity, all of which affect lens design and positioning ${ }^{34}$. Usually the more $\mathrm{O}_{2}$ permeable the plastic, the more fragile the finished lens. The more the fluorine silicone content of the CLmaterial, the more wettable the $\mathrm{CL}^{35}$.

PMMA CLs are occasionally useful, although the practitioner must recognize that this material has virtually no oxygen permeability and that corneal metabolism is totally dependent on tear exchange through the tear pump mechanism. Concern about hypoxia in patients with corneal grafts or previous superficial pannus, possibly from the use of hydrogel CLs of optical power in excess of 10.00D is an indication for the use of RGP $\mathrm{CLs}^{36}$. Dusty environment poses more problems in RGPCL wear.

Scleral or haptic high DK RGP (or even PMMA) CLs can be used in the management of keratoconus or other therapeutic cases such as ocular cicatricial pemphigoid or Steven-Johnson syndrome.

Hybrid and silicone lenses combine aspects of both rigid and flexible lens materials producing lenses like the piggyback systems (wherein a rigid $\mathrm{CL}$ is worn over a hydrogel CL on one eye ${ }^{37,38}$, nonhydrogel flexible materials (e.g. Silsoft ${ }^{39}$ and Softperm $^{40}$ ). Though not in common use such lenses are extremely helpful in rare cases of regular or irregular corneal astigmatism (including keratoconus) or aphakia.

\section{RGP LENSES VERSUS SOFT CONTACT LENSES AND THE OXY GEN ISSUE}

While RGP lenses are recommended in situations of high visual demand, durability, ease of care, extended wear, when cylinder error is greater than sphere, in with the-rule astigmatism and when there is poor visual acuity with soft lenses, the soft contact lenses are preferable in dusty environments, contact sports, occasional wear, sphere error greater than twice the cylinder, against the rule astigmatism and poor comfort with RGPs.

About $33 \%$ of contact lens wear complications are attributable to hypoxia ${ }^{49}$ and therefore the oxygen transmissibility of a contact lens is probably the most important single parameter in terms of maintaining the physiologic integrity of cornea.

This therefore calls for a need for review of the DK data. Bejamin ${ }^{41}$ has divided contact lens materials into the following five categories based on oxygen transmission:

Low: (Dk/L<12); Medium (Dk/L=12- 25); High $(\mathrm{Dk} / \mathrm{L}=26-50)$; Super $(\mathrm{Dk} / \mathrm{L}=51-80)$; and Hyper $(\mathrm{Dk} / \mathrm{L}>80)$. The water content of hydrogel lenses typically limits them to the low and medium oxygen groups, but the tear pump activity also plays a role. While rigid lenses exchange 10 to 20 percent of tears with the blink, the soft lens tear pump exchange as little as one percent. Smaller diameter of RGPs also improves the oxygen availability to the cornea.

Bennett $^{24}$ recommended that we should reserve: Low Dk/L materials for myopic daily wear only, Medium $\mathrm{Dk} / \mathrm{L}$ for myopic and hyperopic daily wears, High Dk/L for myopic flexible wear or hyperopic daily wear, super $\mathrm{Dk} / \mathrm{L}$ for myopic extended wear or hyperopic flexible wear and hyper $\mathrm{Dk} / \mathrm{L}$ for myopic and hyperopic extended wear. These differences in the recommended wearing schedules of myopias and hyperopias is attributable to the differences in the edge and center thicknesses of the minus and plus lenses which brings about differences in their oxygen transmissibility. Increasing the Dk of RGP CLs is often accompanied by compromises in other lens properties vital to the clinical performance of the 
material. High oxygen permeability has frequently been associated with poor surface wetting, increased lens deposit, greater lens flexure and dimensional instabily.

\section{CONCLUSION}

Individuals with refractive errors seek improved visual acuity to enhance their perception and enjoyment of the world. Contact lens, which is a good alternative for the majority of the patients who are motivated to using them. So many years of research and continual advances in contact lens technology have resulted in a good understanding of many of the biocompatibility issues.

Because these lenses float within the tear layer, in intimate contact with the anterior ocular surface, great care should be taken in the prescription and application of contact lenses, and in the supervision of patients who wear them. Infection remains a significant but rare complication of lens wear. Hypoxia appears to contribute most to the complications of contact lens wear. Minimization of extended or overnight wear along with attention to compliance in lens care also reduce the risk of complications associated with CL wear. The fitting objectives of CLs remain to maximize vision, maintain comfort, and minimize interference with corneal function and structure. RGP lenses in addition to providing the cornea with more oxygen are more durable, easier to handle, and require less care than do hydrogels.

\section{R E F E R E N C E S}

1. Bennett, I., Aron, F. (1993): State of the professionï 1993. Optom. Econ, 3:8-14.

2. Barr, J. T., Bailey, N. J. (1991): The development of contact lenses. In: Bennett, E. S., Weissman, B. A., Eds. Clinical Contact Lens Practice, Philadelphia: J.B. Lippincott, 1072pp.

3. Krachmer, J. H. and Purcell, J. J. (1998): Bacterial corneal ulcers in cosmetic soft contact lens wearers. Arch. Ophthalmol, 96:57-6I.

4. Rozenman, Y., Donnenfeld, E. D. and Cohen, E. J. (1989): Contact lens-related deep stromal neovascularization. Am. J. Ophthalmol, 107: 27-32.

5. Schein, O. D., Glynn, R. J. and Poggio, E. D. (1989): The relative risk of ulcerative keratitis among users of daily wear and extended wear soft contact lenses. New Eng. I. J. Med, 321:773-8.

6. Poggio. E. D., Glynn, R. J. and Schein, O. D. (1989): The incidence of ulcerative keratitis among user of daily wear and extended wear soft contact lenses. New Eng. I. J. Med, 321: 779-83.

7. Keech, P. M., Ichikawa, L., Barlow, W. (1996) :Prospective study of contact lens complications in a managed care setting. Optom. Vis. Sci, 73:653-8.

8. Levy, B., McNamara, N., Corzine, J., Abbot R. L. (1997): Prospective trial of daily and extended wear disposable contact lenses. Cornea, 16:274-6.
9. Bruce, A. S., Brennan, N. A., Lindsay, R. (1996): Diagnosis and management of ocular changes during contact lens wear. J. Clin. Signs Ophthalmol, 17(I): 2-11.

10. Westheimer, G (1962): The visual world of the new contact lens wearer. J. Am. Optom. Assoc, 34:135-8.

11. Robertson, D. M., Ogle, K. N. and Diyer, J. A. (1967): Influence of contact lenses on accommodation. Am. J. Ophthalmol，64:86071.

12. Paugh, J. R., Matoba, R. and Matoba, E. N. Y. (1987): Plus acceptance in hard contact lens wearers. Am. J. Optom. Physiol. Opt, 64:7037.

13. Gassett, A. R. and Kaufman, H. E. (1970): Therapeutic uses of hydrophic contact lenses. Am. J. Ophthalmol, 64:703-7.

14. Grosvenor, T., Perrigin, J., Perrigin, D. and Quintero, S. (1990): Silicone acrylate contact lenses for myopia control: 3-year results. Optom. Vis. Sci, 67:764-9.

15. Polse, K. A., Brand, R. J., Vastine, D. W. and Schewalbee, J. S. (1983): Corneal changes accompanying orthokeratology. Plastic or elastic? Results of a randomized clinical trial. Arch. Ophthalmol, 101: 1873-8.

16. McMahon, T. T. and Krefman, R. A. (1989): A four year retrospective study of prosthetic hydrogel lens use. Int. Contact Lens Clin, 11:149-55.

17. Eichenbaum, J. W., Feldstein, M. and Podos, S. M. (1982): Extended wear aphatik soft contact 
lenses and corneal ulcers. Br.J. Ophthalmol, 66:663-6.

18. Maguen, E., Saiz, J. J. and Nesburn, A. B. (1992): Pseudomonas corneal ulcer associated with rigid, gas permeable, daily wear contact lenses in a patient infected with human immunodefiency virus. Am. J. Ophthalmol, 133:336-7.

19. Sarver, M. D. (1972): Vision with hydrophilic contact lenses. J. Am. Optom. Assoc, 43:31620.

20. Snyder, C. and Talley, D. K. (1989): Masking of astigmatism with selected spherical soft contact lenses. J. Am. Optom. Assoc, 60:72831.

21. Thompson, T. T. (1999): FDA Four lens groups. Tyler's Quarterly Soft Contact Lens Parameter Guide, 16: first index page.

22. Brennan, N. A., Efron, N., Weissman, B. A. and Harris, M. G. (1991): Clinical application of oxygen transmissibility of powered contact lenses. CLAOJ, 17:169-72.

23. Fatt, I. and St. Helen, R. (1971): Oxygen tension under an oxygen- permeable contact lens. Am. J. Optom. Arch. Am. Acad. Optom, 48:545-55.

24. Fatt, I. (1978): Gas transmission properties of soft contact lenses. In: Ruben, M., Edn. Soft contact lenses. New York: John Wiley \&Sons, pp83-110.

25. Fatt, I. and Neumann, S. (1989): The average oxygen transmissibility of contact lenses: application of the concept to laboratory measurements, clinical performance and marketing. Neue Oticker J, 31:55-8.

26. Weissman, B. A. and Phan, C. (1992): The in Dk/L. Optom. Vis. Sci, 63:639-44.

27. Alvord, L., Court, J. and Davis, C. (1998): Oxygen permeability of a new type of high Dk soft contact lens material. Optom. Vis. Sci, 75:30-6.

28. Holden, B. A. (1975): The principles and practice of correcting astigmatism with soft contact lenses, Aust. J. Optom, 58:279-99.

29. Hanks, A. J., Weisbarth, R. E. and McNally, J.
J. (1987): Clinical performance comparisons of toric contact lens designs. Int. Cont. Lens Clin, 14:16-21.

30. Weissman, B. A. (1986): Theoretical optics of toric hydrogel contact lenses. Am. J. Optom. Physiol. Opt, 63:536-8.

31. Snyder, C. (1989): A review and discussion of crossed cylinder effects and over- refractions with toric soft contact lenses. Int. Cont. Lens Clin, 16(4):113-8.

32. Businger, U., Treiber, A. and Flury, C. (1989): The etiology and management of three and nine o' clock staining. Int. Cont. Lens Clin, 16:136-9.

33. Refojo, M. F. (1984): The siloxane bond in contact lens materials: effect of methyl and phenyl content on oxygen permeability of silicone lenses. Int. Cont. Lens. Clin, 11:83-6.

34. Weissman, B. A. (1985): Mass of rigid contact lenses. Am. J. Optom. Physiol. Opt, 62:322-8.

35. Guillon, M. and Guillon, J. P. (1988): Pre-Lens tear film characteristics of high Dk rigid gas permeable lens. Am. J. Optom. Physiol. Opt, 65:73-8.

36. Chan, W.K. and Weissman, B. A. (1996): Corneal pannus associated with contact lens wear. Am. J. Ophthalmol, 121:540-6.

37. Baldone, J. A. and Clark, W. B. (1970): Contact lens in the aphatik child. Cont. Lens Med. Bull, 3:25-7.

38. Yeung, K., Eghbali, F., Weissman, B. A. (1995): Clinical experience with piggy back contact lens systems on keratoconic eyes. J.Am. Optom. Assoc, 66:539-43.

39. Nelson, L. B., Cutler S. I. and Calhoun, J. H. (1985): Silsoft extended wear contact lenses in pediatric aphakia Ophthalmol. 92:1529-1531.

40. Maguen, E., Martinez, M. and Rosner, I. R. (1991): The use of Saturn ll lens in keratoconus. CLAOJ, 17:41-43.

41. Benjamin, W. J. (1994): Oxygen transport through contact lenses. Contact Lens Practice. Chapman \& Hall, London, pp44-70.

42. Bennett, E. S. (1998): RGPs: When oxygen is a priority. Contact Lens Spectrum, 13(4):18. 